\title{
SISTER OF TM3 activates FRUITFULL1 to regulate inflorescence branching in tomato
}

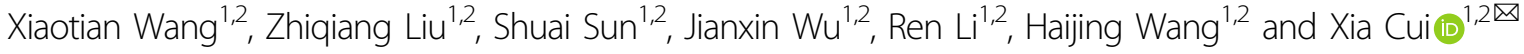

\begin{abstract}
Selection for favorable inflorescence architecture to improve yield is one of the crucial targets in crop breeding. Different tomato varieties require distinct inflorescence-branching structures to enhance productivity. While a few important genes for tomato inflorescence-branching development have been identified, the regulatory mechanism underlying inflorescence branching is still unclear. Here, we confirmed that SISTER OF TM3 (STM3), a homolog of Arabidopsis SOC1, is a major positive regulatory factor of tomato inflorescence architecture by map-based cloning. High expression levels of STM3 underlie the highly inflorescence-branching phenotype in ST024. STM3 is expressed in both vegetative and reproductive meristematic tissues and in leaf primordia and leaves, indicative of its function in flowering time and inflorescence-branching development. Transcriptome analysis shows that several floral development-related genes are affected by STM3 mutation. Among them, FRUITFULL1 (FUL1) is downregulated in stm3 $\mathrm{cr}$ mutants, and its promoter is bound by STM3 by ChIP-qPCR analysis. EMSA and dual-luciferase reporter assays further confirmed that STM3 could directly bind the promoter region to activate FUL1 expression. Mutation of FUL1 could partially restore inflorescence-branching phenotypes caused by high STM3 expression in ST024. Our findings provide insights into the molecular and genetic mechanisms underlying inflorescence development in tomato.
\end{abstract}

\section{Introduction}

Higher plants exhibit various inflorescence architectures progressing in complexity from a solitary flower to complex structures that contain multiple branches and flowers. The architecture of inflorescences is one of the determinant traits for many crops, such as rice (Oryza sativa), maize (Zea mays), and tomato (Solanum lycopersicum) ${ }^{1}$. Favorable inflorescence branching is always a major breeding target for achieving desirable production by balancing the sink-source relationship. Distinguished from the raceme-type inflorescences of Arabidopsis and panicletype inflorescences of rice, tomato has a cyme-type inflorescence lacking a main axis, which initiates from a sympodial inflorescence meristem (SIM). The SIM

\footnotetext{
Correspondence: Xia Cui (cuixia@caas.cn)

${ }^{1}$ Key Laboratory of Biology and Genetic Improvement of Horticultural Crops of the Ministry of Agriculture, Institute of Vegetables and Flowers, Chinese

Academy of Agricultural Sciences, Beijing 100081, China

${ }^{2}$ Sino-Dutch Joint Laboratory of Horticultural Genomics, Institute of Vegetables and Flowers, Chinese Academy of Agricultural Sciences, Beijing 100081, China
}

generates a new SIM before terminating in a floral meristem (FM) and reiteration of this pattern produces tomato inflorescences and determination of branching ${ }^{1-4}$.

A series of regulatory genes that have received much attention makes major contributions to inflorescence architecture in tomato by changing the inflorescencebranching pattern. A larger vegetative shoot apical meristem (SAM) often produces more branching inflorescences. The WUSCHEL-CLAVATA (WUS-CLV) feedback regulatory loop is conserved maintaining the balance among SAM activities and controlling meristem size in Arabidopsis, rice, tomato, and other plants ${ }^{5-11}$. Mutations in the CLV pathway genes, SICLV3, FASCIATED AND BRANCHED (FAB), and FASCIATED INFLORESCENCE (FIN), cause meristems to enlarge, leading to an increase in inflorescence branching in tomato ${ }^{12,13}$.

During phase transitions, flowering time genes are also important players in regulating inflorescence architecture in tomato. The florigen mutant, single-flower truss (sft), is late-flowering and disrupts normal tomato sympodial

\section{(c) The Author(s) 2021}

(c) Open Access This article is licensed under a Creative Commons Attribution 4.0 International License, which permits use, sharing, adaptation, distribution and reproduction cc) in any medium or format, as long as you give appropriate credit to the original author(s) and the source, provide a link to the Creative Commons license, and indicate if changes were made. The images or other third party material in this article are included in the article's Creative Commons license, unless indicated otherwise in a credit line to the material. If material is not included in the article's Creative Commons license and your intended use is not permitted by statutory regulation or exceeds the permitted use, you will need to obtain permission directly from the copyright holder. To view a copy of this license, visit http://creativecommons.org/licenses/by/4.0/. 
growth, which reverses the inflorescence toward vegetative functioning after the initiation of one or a few flowers depending on the growing conditions ${ }^{14-16}$. The jointless (j) mutant produces indeterminate inflorescences that revert to vegetative growth after the production of two or three flowers ${ }^{16,17}$. Moreover, J and SFT cooperatively regulate the architecture of inflorescences preventing early changes in inflorescence meristem (IM) identity once inflorescence morphogenesis is initiated ${ }^{18}$. In addition, FALSIFLORA (FA), the tomato ortholog LEAFY, controls flowering time and floral meristem identity. The $F A$ mutation results in the conversion of flowers into secondary shoots and produces a highly branched inflorescence $^{19,20}$. In addition to these promotion-flowering genes, TERMINATING FLOWER (TMF) encodes an ALOG family protein and affects inflorescence organization in tomato. The tmf mutants flower early and convert multiflowered inflorescence into a solitary flower ${ }^{21}$. TMF and SIBOPs act synergistically to prevent precocious flowering and promote inflorescence complexity. All inflorescences on the slbop $1 / 2 / 3$ triple mutant develop only one or two flowers ${ }^{22}$.

In addition, floral meristem identity genes have a profound influence on tomato inflorescence architecture. Loss of ANANTHA (AN) and COMPOUND INFLORESCENCE (S) delay the progression of an IM to the floral meristem (FM), resulting in additional branching ${ }^{3,23}$. JOINTLESS2 (J2), ENHANCER-OF-JOINTLESS2 (EJ2) and LONG INFLORESCENCE (LIN), three SEPALLATA 4 (SEP4) proteins, have redundant roles in inflorescence branching and cause a quantitative range of inflorescence branching with combinations of homozygous and heterozygous $J 2$ and $E J 2$ mutations in tomato ${ }^{24-26}$. However, reconfiguration of inflorescence branching for breeding higher yield varieties in tomato remains challenging due to the limited known loci or genes and the largely unknown underlying mechanisms.

SUPPRESSOR OF OVEREXPRESSION OF CONSTANS 1 (SOC1) was first identified as an important flowering time integrator that regulates the floral transition in Arabidopsis ${ }^{27-29}$. In addition, SOC1 and three MADS-box transcription factors, SHORT VEGETATIVE PHASE (SVP), AGAMOUS-LIKE 24 (AGL24), and SEP4, act redundantly and directly suppress TERMINAL FLOWER 1 (TFL1) to control Arabidopsis inflorescence architecture $^{30}$. STM3, a homolog of SOC1 in tomato, also regulates inflorescence branching ${ }^{26}$. However, the mechanism by which STM3 positively regulates inflorescence branching in tomato is still unknown. In our study, we confirmed that STM3 is a major positive factor controlling inflorescence branching in tomato. Mutation of STM3 reduced the number of inflorescence branches, and overexpression of STM3 produced compound inflorescences. STM3 directly binds the promoter of
FRUITFULL1 (FUL1) and activates its expression in vitro and in vivo. Mutation of FUL1 could partially restore inflorescence-branching phenotypes caused by high STM3 expression. Our findings provide insights into the molecular and genetic mechanisms underlying inflorescence development in tomato.

\section{Results \\ qMIB1 controls inflorescence branching in tomato}

To identify the loci regulating tomato inflorescence branching, we found a line, ST024, which has extraordinary inflorescence branching with long sepals and jointless pedicels that are similar to the $j 2$ and ej2 mutants ${ }^{24,31}$, from a set of 201 stable recombinant inbred lines (RILs) generated from a cross of Solanum Lycopersicum var. cerasiforme LA1310 (CC) and S. Lycopersicum Moneymaker $(\mathrm{MM})^{32}$. The parental $\mathrm{CC}$ was generally a simple inflorescence, and less than $20 \%$ of inflorescences had two branches. Compared with $\mathrm{CC}$, MM showed complex inflorescence branching, and more than $60 \%$ of inflorescences had more than two branches (Fig. 1a; Supplementary Fig. S1a-c). The genome sequencing data of ST024 and genotyping revealed a 5 bp deletion in the $4^{\text {th }}$ exon of $J 2$ leading to early termination of its translation, which was named $j 2^{d e l}$, and a $564 \mathrm{bp}$ insertion in the $5^{\text {th }}$ intron of $E J 2$, similar to the previously reported weak $e j 2^{W}$ mutant ${ }^{24}$ that resulted in a jointless pedicel and long sepal of ST024 (Supplementary Fig. S1d-f). Based on RIL population sequencing data, we screened ten lines with the same $j 2^{d e l}{ }^{\text {ej }} 2^{W}$ genotypes ${ }^{32}$, unexpectedly, these RILs with the same $j 2^{\text {del }} e j 2^{W}$ genotypes showed different inflorescence architectures (Supplementary Fig. S1g), suggesting that additional variants influence inflorescence diversity in RILs.

To explore the genetic basis of the branch discrepancy among these RILs, we crossed ST024 with ST147, one of the RILs with the $j 2^{d e l} e j 2^{W}$ genotype, and a weakly branched inflorescence (Fig. 1b). We sequenced pools of DNA from two subcategorized groups of the $F_{2}$ population that exhibited extreme inflorescence architecture. One locus, qMIB1 (QTL for MULTIPLE INFLORESCENCE $B R A N C H$ 1), on chromosome 1 was identified (Supplementary Fig. S2a). To further investigate the contribution of $q M I B 1$, we generated NILs by crossing ST147 and ST024 and selected offspring heterozygous at the qMIB1 locus. After six generations of selfing, $F_{7}$ with a $191 \mathrm{~kb}$ heterozygous region around $q M I B 1$ was obtained. The progenies with different genotypes in this region were defined as near-isogenic lines (NILs) by marker screening. We compared inflorescence-branching number among NIL-mib1 ${ }^{\mathrm{MM}}$, NIL-MIB1 $1^{\mathrm{CC}}$, and NIL-MIB1 ${ }^{\mathrm{CM}}$ and found that NIL-mib1 $1^{\mathrm{MM}}$ plants had significantly more branches than NIL-MIB1 ${ }^{\mathrm{CC}}$ plants, and the heterozygous NIL$M I B 1^{\mathrm{CM}}$ line showed an intermediate extent of 
a

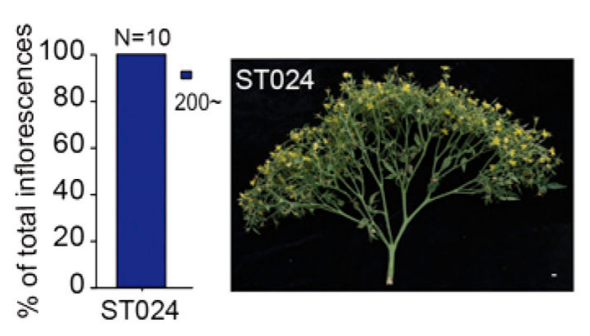

b

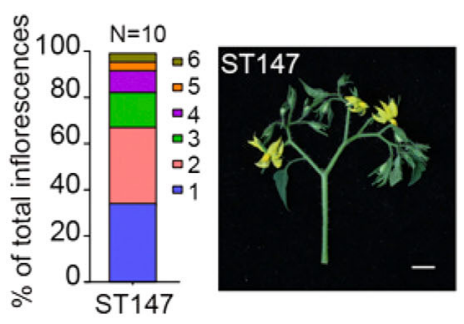

C
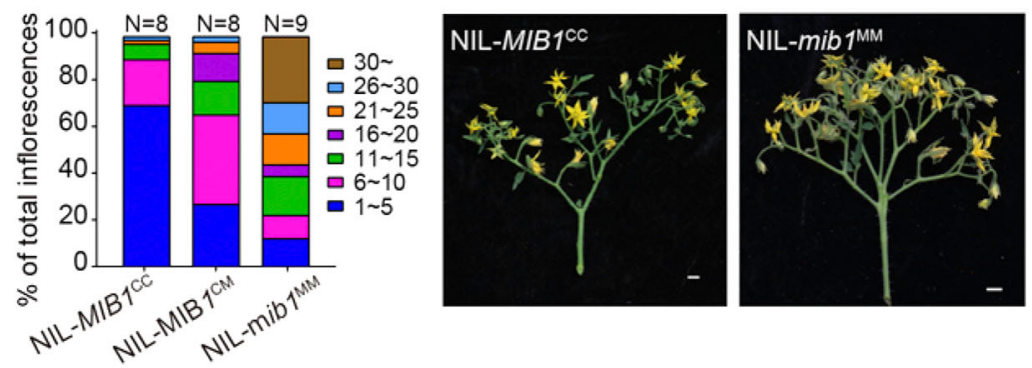

d
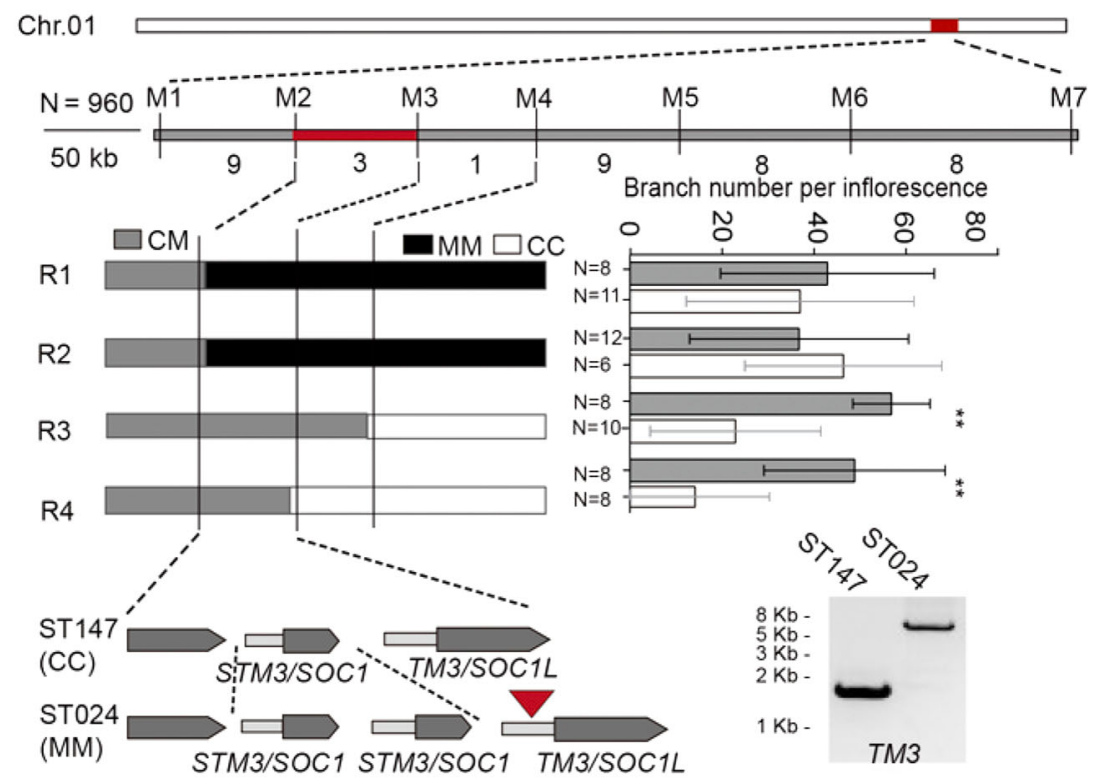

e

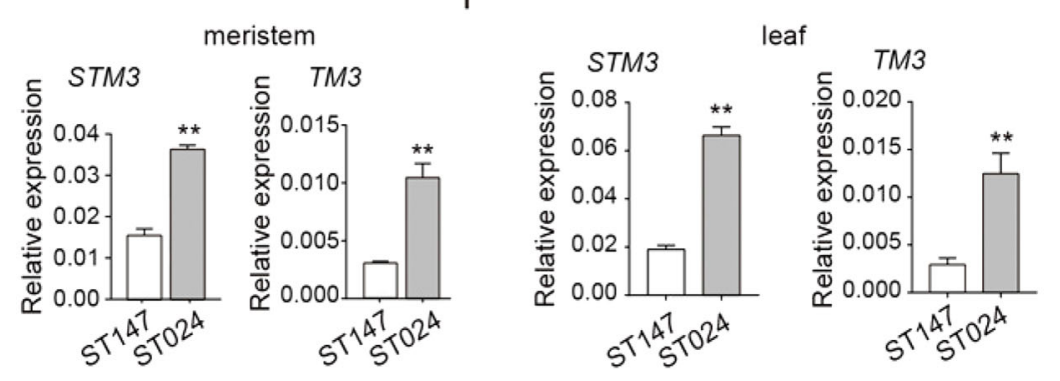

Fig. 1 (See legend on next page.) 
(see figure on previous page)

Fig. 1 Identification and Characterization of qMIB1. a, b Quantification of inflorescence branching and representative images from the two parent lines: ST024 (a) and ST147 (b). c The phenotype of near-isogenic lines. NIL-MIB $1^{C C}$ and NIL-mib $1^{\text {MM }}$ represent the ST147 and ST024 genotypes, respectively, and CM is a heterozygous genotype. $\mathbf{d}$ Fine mapping of qMIB1. Top panel, positional cloning narrowed qMIB1 to the DNA segment between markers M2 and M3. The numbers below the bars indicate the number of recombinants. Middle panel, high-resolution mapping of qMIB1 (left), and the progeny test of four recombinants (right). The black, gray, and white boxes indicate chromosome regions with the MM, CM, and CC genotypes, respectively. N, plant number. Bottom panel, genomic diagram showing the candidate ORFs. The gel picture showed a large insertion in the TM3 promoter of ST024. e, f Quantification of the relative expression of STM3 and TM3 in reproductive meristematic tissues and leaves of 21-dold seedlings between ST147 and ST024. UBIQUITIN3 was used as the internal control. Data were compared by two-tailed Student's $t$-test, ${ }^{* *} P<0.01$, error bar, SD

inflorescence branching that is consistent with $\mathrm{F}_{1}$ hybrids (Fig. 1c; Supplementary Fig. S2b, c), indicating that $q M I B 1$ is partially dominant.

\section{Fine mapping of $q M I B 1$}

Using 960 individuals of the $\mathrm{F}_{2}$ population, $q M I B 1$ was finally narrowed to a $76.12 \mathrm{~kb}$ region containing three annotated genes: Solyc01g091950 and two neighboring MADS-box genes, Solyc01g092950 (STM3) and Solyc01g093965 (TM3) (Fig. 1d; Supplementary Fig. S3a). We obtained the sequence of the STM3 transcript (Supplementary Fig. S3b, c) by RACE and found no variations in the coding regions of these two genes between ST024 and ST147. The sequence data for these lines and our PCR results revealed an $\sim 20 \mathrm{~kb}$ repeat sequence at this region in the ST024 genome but not in ST147, which is consistent with a recent study that demonstrated that structural variation in the tomato genome resulted in an additional copy of $S T M 3^{26}$. In addition, we found one gap in the promoter of TM3 according to the SL2.50 reference genome. Using two specific primers on the two sides of the gap, an $~ 7 \mathrm{~kb}$ DNA fragment was amplified in ST024/ MM, but only a $1.7 \mathrm{~kb}$ DNA fragment was obtained in ST147/CC (Fig. 1d and Supplementary Fig. S4a), suggesting that the gap was caused by an $\sim 5.3 \mathrm{~kb}$ DNA fragment insertion. To test whether the repeat and insertion affect STM3 or TM3 expression, we detected the expression levels of STM3 and TM3 in reproductive meristematic tissues and leaves of 21-d-old seedlings by quantitative real-time PCR (qRT-PCR) and found that the expression levels of both STM3 and TM3 were higher in ST024 than in ST147. Higher expression levels were also detected in NIL-mib1 ${ }^{\mathrm{MM}}$ than in NIL-MIB1 ${ }^{\mathrm{CC}}$ (Fig. 1e, f; Supplementary Fig. S4b, c). These results suggested that high expression of STM3 and TM3 caused by these sequence variations may underlie the more inflorescencebranching phenotype in ST024.

\section{STM3 and TM3 redundantly regulate inflorescence branching in tomato}

To verify that the higher expression of STM3 and TM3 led to more inflorescence branching in ST024, we transformed ST147 with vectors carrying the full-length CDS of TM3 and STM3 fused with YFP-HA or GFPFLAG tags, respectively, and driven by the $35 \mathrm{~S}$ promoter. Two independent overexpressed transgenic lines for each construct were obtained (Fig. 2a). As expected, the STM3OE transgenic plants exhibited higher branching inflorescence than ST147 but were still far less than ST024, whereas the TM3OE lines had no obvious differences from ST147 (Fig. 2b, c). In addition, we also generated single null mutants, stm $3 c r-1, t m 3 c r-1$, and $t m 3 c r-2$, and the null double mutants stm $3 t m 3 c r-1$ and stm 3 tm3cr-2 using CRISPR/Cas9 in the ST024 background (Fig. 2d). Intriguingly, reduced inflorescence branching was observed in all single and double mutants; yet stm3cr single and stm $3 \mathrm{tm} 3 \mathrm{cr}$ double mutants had strongly suppressed branching, tm3cr mutant had weakly suppressed branching (Fig. 2e, f). These results suggested that STM3 and TM3 may have at least partially redundant functions in controlling inflorescence-branching development.

It should also be noted that only the $s t m 3 \mathrm{tm} 3 \mathrm{cr}$ and stm3cr plants photocopied a late-flowering phenotype, which is consistent with a recent study that demonstrated a delayed flowering phenotype in lines with the $s b 1^{C R-1}$ and $s b 1^{C R \text {-del }}$ mutants ${ }^{26}$ (Supplementary Fig. S5), but there was no obvious change in $t m 3 c r$ compared with ST024.

\section{STM3 expressed in tomato different meristems and tissues}

To better understand the individual and combined roles of tomato STM3 and TM3, we first analyzed their expression patterns in different organs. Both genes were expressed in roots, flowers, and meristems, with especially strong expression in leaves (Fig. 3a), an expression profile consistent with SOC1 from Arabidopsis ${ }^{33}$. Moreover, in situ hybridization analysis of longitudinal sections of various meristems showed that STM3 and TM3 exhibit similar expression profiles, with strong accumulation at apices of meristems, and both accumulated in leaf primordia and vascular bundles (Fig. 3b), supporting their roles in inflorescence development. Without exception, the expression level of STM3 was higher than that of TM3 in all tissues, which is in line with their functions in inflorescence architecture and flowering. 
a
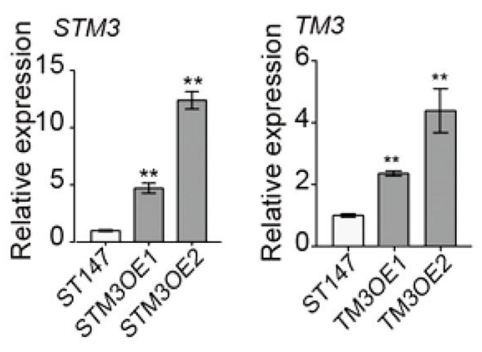

C
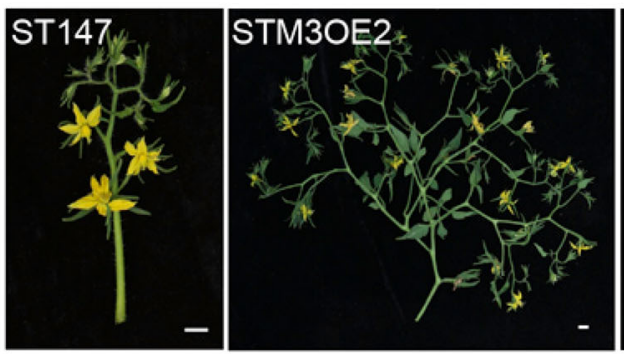

d

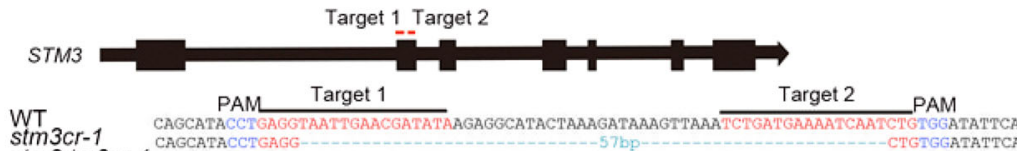

$s t m 3$
$\mathrm{~s} t \mathrm{tm} 3 \mathrm{t} 3 \mathrm{cr}-1$ CAGCATACCTGAG--.-TTGAACGATATAAGAGGCATACTAAAGATAAAGTTAAATCTGATGAAAATCAATCTGTGGATATTCA

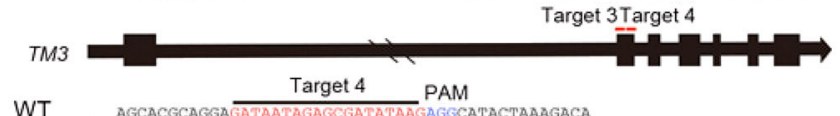

WT

TAAAGACA

tm3cr-2 AGCACGCAGGAGATATATAGAGGGGATATATAMAGAGGGCATACTAAAGGACA

WT

PAM Target 3

stm3 tm 3cr-2 CAGAGTTCAACCTGAAAACCAAGC--10bP-- TATCTACAG

e

f
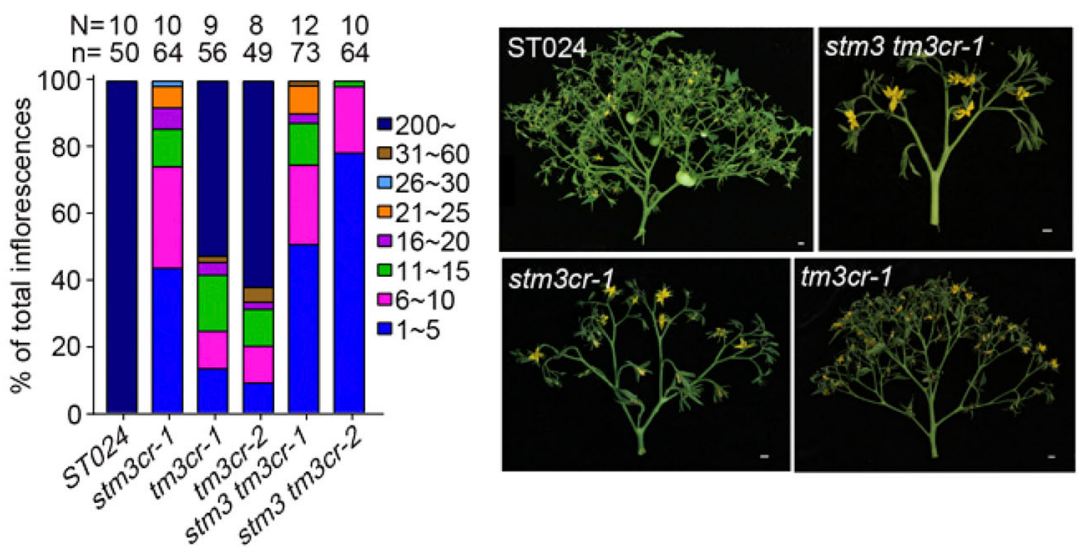

Fig. 2 (See legend on next page.) 
(see figure on previous page)

Fig. 2 STM3 and TM3 function redundantly to control inflorescence branching in tomato. a Expression levels of STM3 and TM3 in overexpressed transgenic plants. b Quantification of inflorescence branching in ST147 and overexpressed transgenic plants. c Representative inflorescence images of ST147 and overexpressed transgenic plants. d STM3 and TM3 mutations generated by CRISPR/Cas9. Red words mark the position of targets. Blue words indicate the protospacer-adjacent motif (PAM). Green words mark the mutation sequences of one $s t m 3 \mathrm{cr}-1$ mutant, two $\mathrm{tm} 3 \mathrm{cr}$ mutants, and two $\mathrm{stm} 3 \mathrm{tm} 3 \mathrm{cr}$ mutants in ST024. e Quantification of inflorescence branching in ST024, the single and double mutants. f Representative inflorescence images of all mutants. $\mathrm{N}$, plant number, $\mathrm{n}$, inflorescence number, scale bars $=1 \mathrm{~cm}$

a

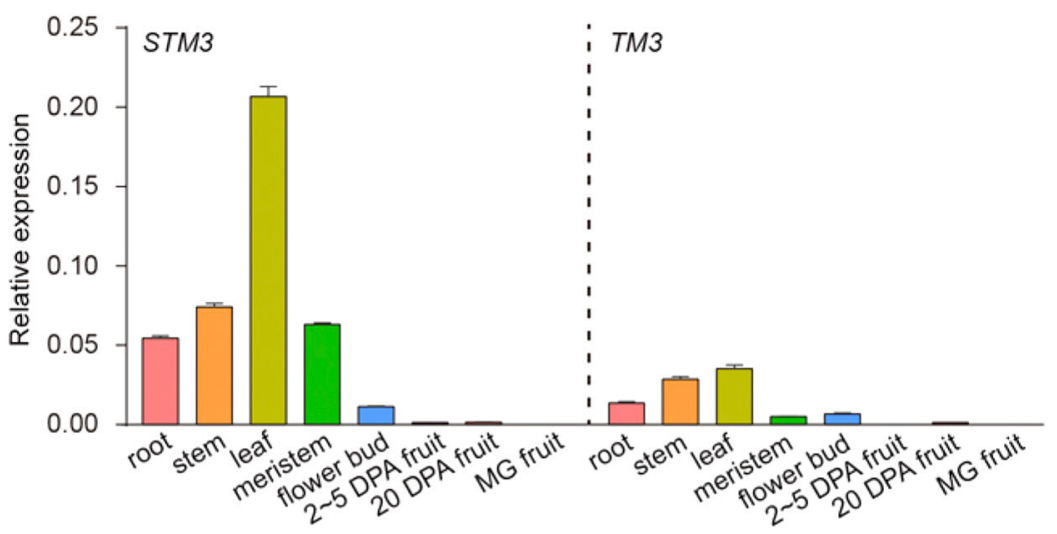

b

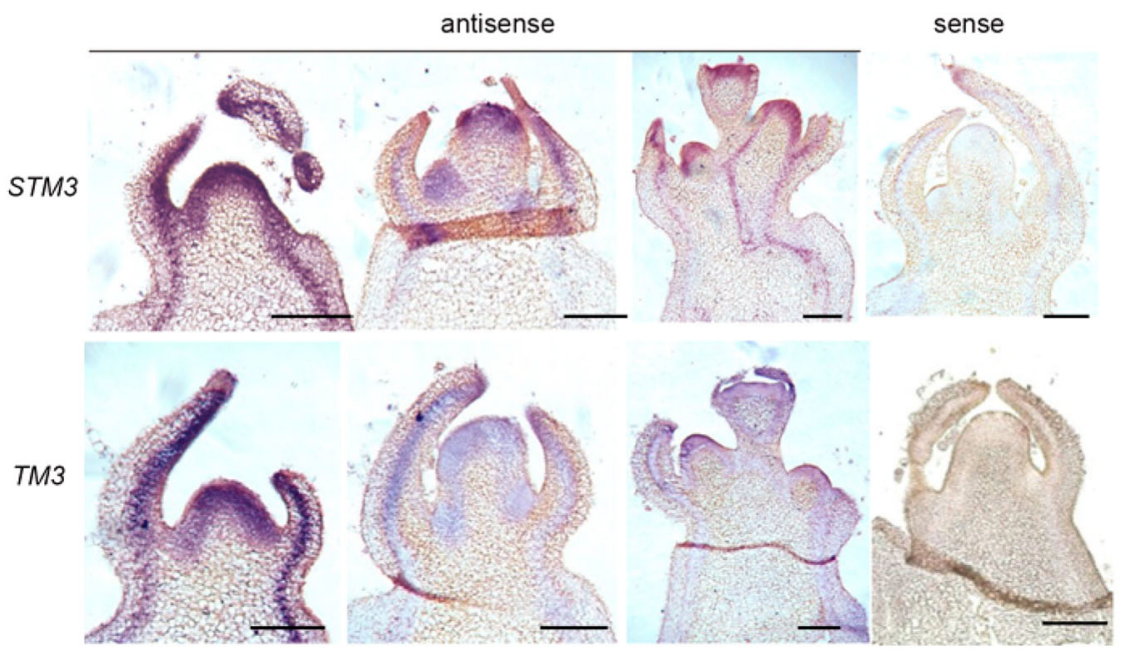

Fig. 3 STM3 is expressed in vegetable and reproductive meristems. a qRT-PCR for STM3 and TM3 expression in root, stem, leaf, meristem, flower, and fruit. The root, stem, leaf, and meristem are from 30-d-old seedlings. DPA, days post anthesis. MG mature green. UBIQUTIN3 was used as the internal control. Error bar, SD. b In situ mRNA hybridization of STM3 and TM3 mRNA transcripts in vegetative and reproductive meristems of MM. Scale bars $=100 \mu \mathrm{m}$

\section{STM3 physically interacts with TM3}

MADS-box proteins often constitute multimeric complexes to direct a variety of developmental processes ${ }^{34}$. Therefore, we tested the interaction between STM3 and TM3 by yeast two-hybrid assay. Our results indicated that STM3 was able to interact with TM3 and itself (Fig. 4a). Further co-immunoprecipitation (Co-IP) assays using tobacco (Nicotiana benthamiana) leaves, in which STM3FLAG was transiently expressed together with YFP-HA, STM3-YFP-HA, and TM3-YFP-HA, confirmed that STM3 could be immunoprecipitated by STM3-YFP-HA and TM3-YFP-HA fusion proteins but not by the YFP-HA control (Fig. 4b). These results further indicated that STM3 and TM3 control inflorescence architecture together. 
a

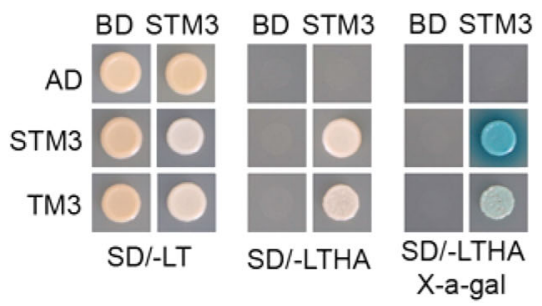

b

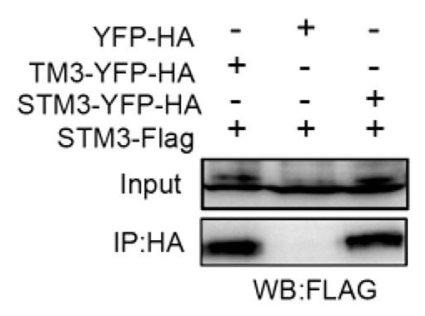

Fig. 4 STM3 interacts with TM3 and itself. a Interaction assays for the STM3 and TM3 proteins in yeast cells. Yeast cells grown on a selective medium (-LTHA) showed a positive interaction. -LT, without Leu and Trp. -LTHA, without Leu, Trp, His, and Ade. b Co-immunoprecipitation assays to examine interactions between STM3 and TM3 in N. benthamiana leaves. Immunoblots of the total protein extracts (Input) and the IP product were performed using an anti-FLAG antibody (a-FLAG)

\section{STM3 regulates multiple biological processes during inflorescence development}

To investigate the molecular mechanism of STM3 in the regulation of inflorescence development, we collected reproductive meristematic tissues of ST024, tm $3 \mathrm{cr}-1$, stm $3 \mathrm{cr}-1$, and stm $3 \mathrm{tm} 3 \mathrm{cr}-1$ to perform RNA-seq analysis with three biological replicates. Here, approximately 30 million uniquely mapped reads were generated for each sample (Supplementary Table S1). The principal component analysis (PCA) results showed that the sequencing data were highly reproducible (Fig. 5a). From the PCA, the stm $3 c r-1$ and stm 3 tm $3 c r-1$ mutants were located closely, which is in a sense consistent with the inflorescencebranching phenotype. In total, compared with ST024, 629, 844 , and 835 differentially expressed genes (DEGs) were identified in $t m 3 c r-1, s t m 3 c r-1$, and stm3 tm3cr-1, respectively (Fig. 5b). We performed Gene Ontology (GO) term enrichment analysis using DAVID to gain insight into the functions of these DEGs between ST024 and the single and double mutants (Supplementary Table S2). In particular, flower development-related, cell differentiation, transcription-related, and maintenance of inflorescence and floral meristem identity terms were enriched in the downregulated genes (Fig. 5c; Supplementary Figs. S6-8). In contrast to the downregulated genes, the development-related process was rarely enriched in the upregulated genes in the single and double mutants, but most genes related to biotic or abiotic response pathways were enriched (Supplementary Figs. S6-8). We further analyzed these 136 downregulated DEGs, which were repressed in any two or more mutants. Among these genes, FUL1 (Solyc06g069430), APETALA1/AP1 (Solyc02g089210 MACROCALYX/MC (Solyc05g056620), SEPALLATA2/SEP2 (Solyc02g089200), and SEPALLATA3/SEP3 (Solyc05g015750), whose Arabidopsis homologs are related to floral meristem establishment, showed reduced expression levels in the single and double mutants $^{35}$ (Fig. 5d). Consistent with the RNA-seq results, our qRT-PCR assays confirmed the differential expression of these genes (Fig. 5e), suggesting that STM3 may regulate inflorescence development through interactions with other developmental regulators.

\section{STM3 targets FUL1 to activate its expression}

FUL1 is the tomato homolog of Arabidopsis FUL, which has been demonstrated to regulate inflorescence architecture in Arabidopsis ${ }^{36-39}$. In tomato, the FUL1 transcript accumulates gradually from the vegetable to reproductive stages and reaches the highest level at the floral meristem ${ }^{23}$, implying that it functions in inflorescence development. In contrast to its reduced expression in the $s t m 3 c r-1$ and stm $3 t m 3 c r-1$ mutants, the expression level of FUL1 was enhanced in the NIL-mib1 ${ }^{\mathrm{MM}}$ plants and STM3OE lines, in which STM3 was highly expressed, compared with NIL-MIB1 ${ }^{\mathrm{CC}}$ and ST147 plants (Fig. 6a). Therefore, FUL1 should be one of the downstream genes of STM3.

As transcription factors, MADS-box proteins could directly bind to gene promoters to regulate their transcription. Similar to other MADS-box proteins, Arabidopsis SOC1 recognizes the CArG box to directly bind its targets $^{28,29}$. To explore whether FUL1 is bound by STM3, we analyzed the promoter sequence of tomato FUL1, and three putative SOC1-binding motifs (S1, S2, and S3) were identified on the FUL1 promoter by JASPAR ${ }^{40}$ (Fig. 6b). We further performed chromatin immunoprecipitation (ChIP) using an HA antibody against the HA-tagged STM3 protein in 35S::STM3-YFP-HA transgenic plants. Obvious enrichment was observed in P4 around S1 but not in the other regions of the FUL1 promoter by ChIPqPCR analysis (Fig. 6c), indicating that STM3 directly targets FUL1 in tomato. To determine whether STM3 can directly bind the motif, we performed electrophoretic mobility shift assays (EMSAs) using a $49 \mathrm{bp}$ DNA fragment of the FUL1 promoter containing $\mathrm{S} 1$ and the recombinant protein of STM3 with an MBP tag at the $\mathrm{N}$-terminus (MBP-STM3). We found that MBP-STM3 but not MBP bound the FUL1 probe. In addition, the 
a

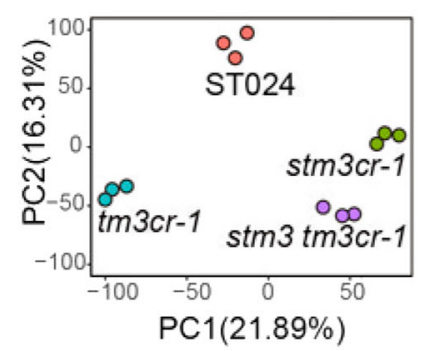

b

\author{
Up-regulated genes Down-regulated genes
}

stm3cr-1 vs ST024 tm3cr-1 vs ST024 stm3cr-1 vs ST024 tm3cr-1 vs ST024

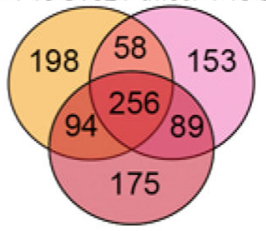

$\operatorname{stm} 3 \mathrm{tm} 3 \mathrm{cr}-1$ vs ST024

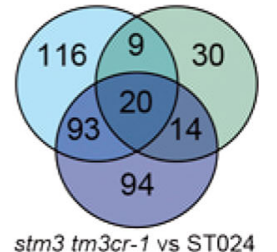

$\operatorname{stm} 3 \operatorname{tm} 3 \mathrm{cr}-1$ vs ST024

C

\section{Biological Process $\mathrm{GO}$ analysis of down-regulated genes in all mutants}

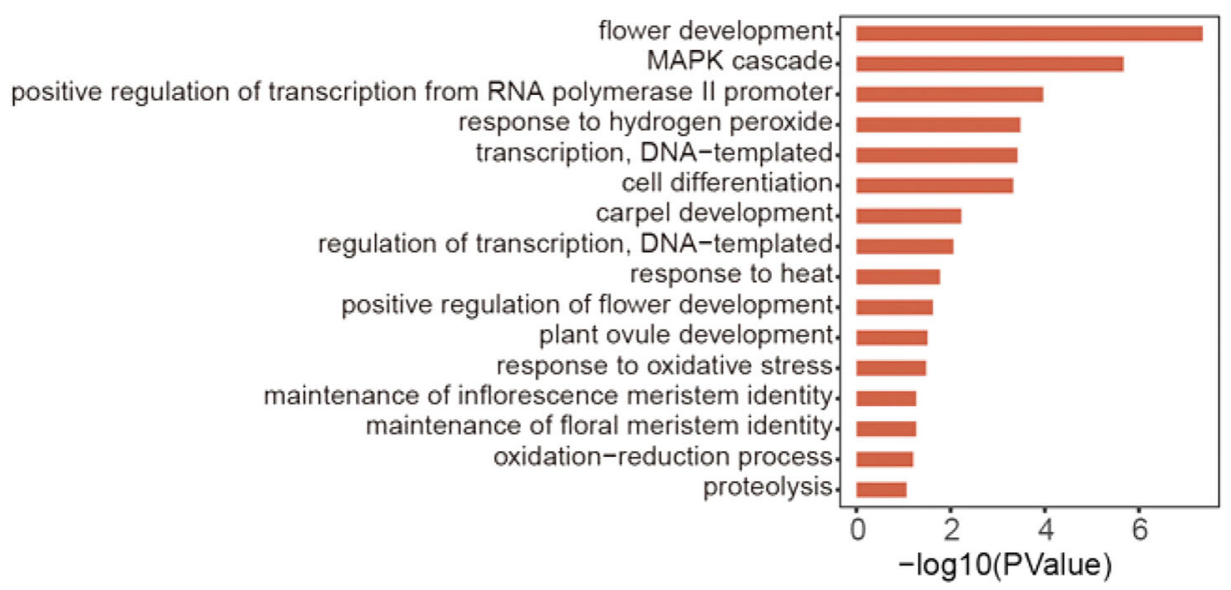

d

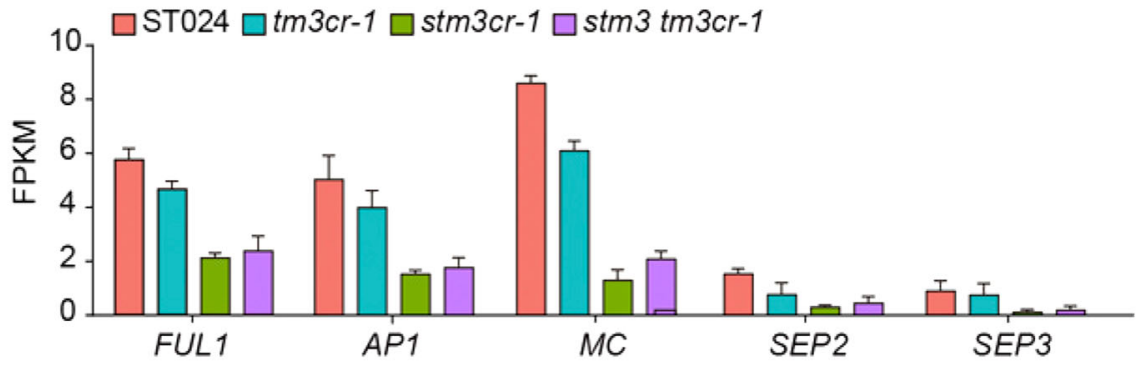

e

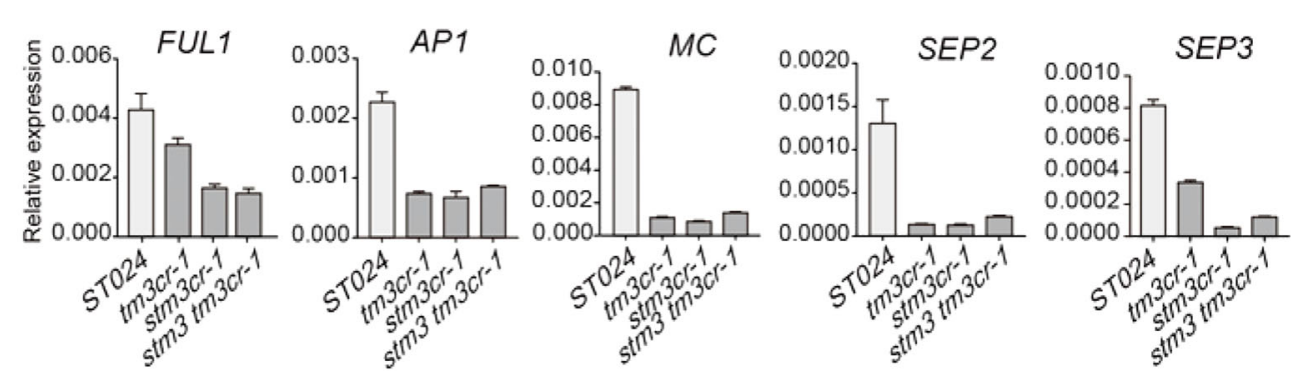

Fig. 5 STM3 regulates multiple biological processes during inflorescence development. a Principal component analysis (PCA) of RNA-seq data. $\mathbf{b}$ Venn diagram showing differentially expressed genes among ST024, tm3 $\mathrm{cr}-1$, stm3 $3 \mathrm{cr}-1$, and $\operatorname{stm} 3 \mathrm{tm} 3 \mathrm{cr}-1$. c GO enrichment analysis of downregulated genes in

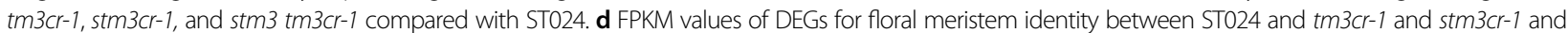
stm3 tm3cr-1. e qRT-PCR verification of DEGs identified by RNA-seq analysis. Data were compared by two-tailed Student's $t$-test, ${ }^{* *} P<0.01$, error bar, SD. 
a

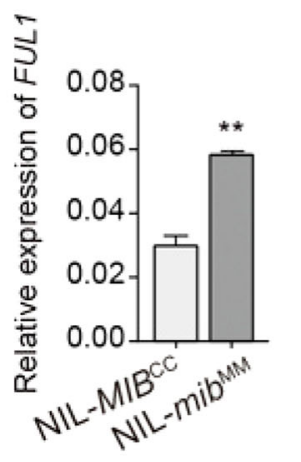

C

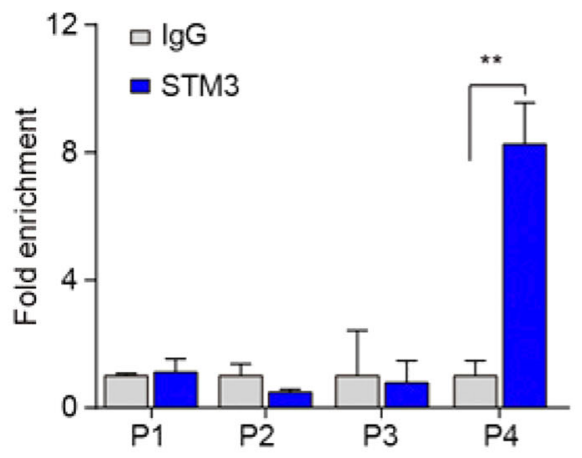

b

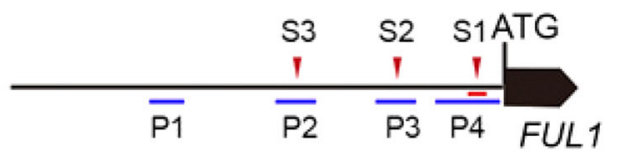

1 motif - Probe used in EMSA

- $100 \mathrm{bp} \quad-$ ChIP-qPCR

e
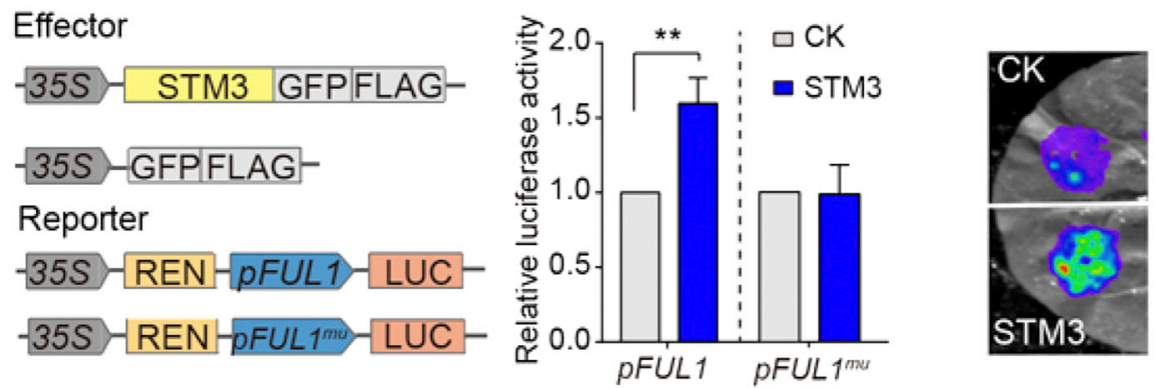

cps

15676

12804

9933

7061

4189

1318

Fig. 6 STM3 directly activates the expression of FUL1. a Relative expression of FUL1 in reproductive meristematic tissues of NIL-MIB ${ }^{C C}$, NIL$\mathrm{mib}_{1} \mathrm{MM}$, and two STM3OE lines. b Schematic diagram of the promoters of FUL1. Red triangles indicate the SOC1-binding motif. Blue lines indicate primers used for ChIP-qPCR assay, and short red lines indicate DNA probes used for EMSA. The translational start site (ATG) is shown at position +1. c ChIP-qPCR assays showing the enrichment of STM3 at the promoter of FUL1. The immunoprecipitated chromatin was analyzed by qPCR using gene-specific primers as indicated in (b), and $\lg G$ was used as the negative control. The intergenic region around ACTIN was used as an internal control. Error bars represent the SD of three biological replicates. Data were compared by two-tailed Student's $t$-test, ${ }^{* *} P<0.01$, error bar, SD. d EMSA showing that STM3 interacts with the probe containing the CArG box on the FUL1 promoter. e Dual-luciferase reporter assay showing that STM3 activates FUL1 expression in tobacco leaves. Fold changes of relative luciferase activity are shown; mu, mutated. Data were compared by two-tailed paired Student's t-test, ${ }^{* *} P<0.01$, error bar, SEM.

excess unlabeled oligonucleotide probe can compete with the labeled probe, and with the increased content of the competed unlabeled oligonucleotide probe, fewer labeled probes are bound by STM3. Moreover, when the CArG box motif of the probe was mutated, the binding of STM3 was obviously reduced (Fig. 6d). All results showed that STM3 could directly bind the FUL1 promoter by recognizing the CArG box. 
To test how STM3 regulates FUL1 expression, we performed a transient expression assay by coexpressing STM3 and the FUL1 promoter in tobacco. Significantly increased luminescence intensity was observed upon coexpression of the STM3 and FUL1 promoters in tobacco leaves compared with the control upon coexpression of GFP-FLAG and the FUL1 promoter. However, no significant differences were observed when coexpressed STM3 and mutated FUL1 promoter (Fig. 6e). In line with the decreased expression level of FUL1 in the stm3cr-1 mutants, these results further confirmed that STM3 activates FUL1 transcription in vivo by binding the CArG box.

\section{FUL1, as a target of STM3, regulates inflorescence branching in tomato}

To further characterize the role of FUL in tomato inflorescence development, we used CRISPR/Cas9 to engineer FUL1 in ST024 plants. Two null alleles, ful1cr-1 and fullcr-2, with $1 \mathrm{bp}$ insertion and $1 \mathrm{bp}$ deletion, were obtained (Fig. 7a). Compared with ST024 plants, the ful1cr-1 and ful1cr-2 engineered plants exhibited reduced inflorescence branching. However, their inflorescence architectures were still more complicated than those of ST147 and stm3cr-1 plants in the ST024 background, indicating that knocking out FUL1 only partially suppressed inflorescence branching (Fig. 7b, c). These results suggested that STM3 regulates inflorescence development to a certain extent through its target, FUL1.

\section{Discussion}

Similar to other plants, tomato inflorescence architecture is governed by inflorescence meristems and their derived meristems between maintenance of indeterminacy and commitment to floral fate ${ }^{4}$. Our studies reveal that TM3 and STM3, two homologs of Arabidopsis SOC1, redundantly regulate tomato inflorescence branching, which is similar to a recent study reporting that natural variation in STM3 causes its higher expression, leading to higher inflorescence branching in tomato $^{26}$. Although TM3 has a redundant function with STM3 in inflorescence development, TM3 overexpression alone could not induce more inflorescence branching in ST147, and the inflorescence architecture of the $t m 3 \mathrm{cr}$ mutant was weaker than that of the $s t m 3 c r$ mutant, suggesting that STM3 is a major gene controlling inflorescence branching in tomato. Consistent with their functions, the expression level of STM3 was obviously higher than that of TM3 in all tissues, although they showed similar expression patterns. Moreover, our results also indicated that SOC1s promote inflorescence branching in a dose-dependent manner in tomato. The moderate branching of $F_{1}$ plants and the various branched phenotypes of the STM3 overexpression transgenic plants were positively related to the expression levels of STM3.

SOC1 is an important activator integrating floral inductive signals from multiple pathways to promote flowering in many plants ${ }^{27,41-44}$. In contrast to only one

a

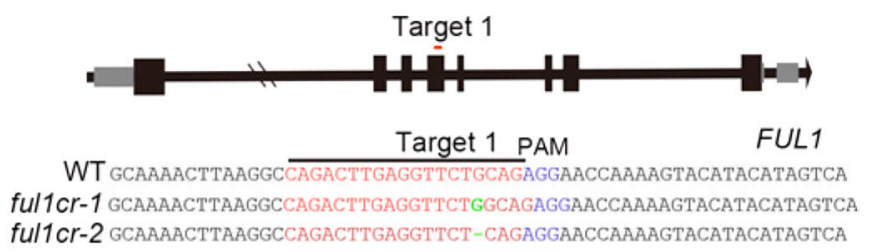

b

C
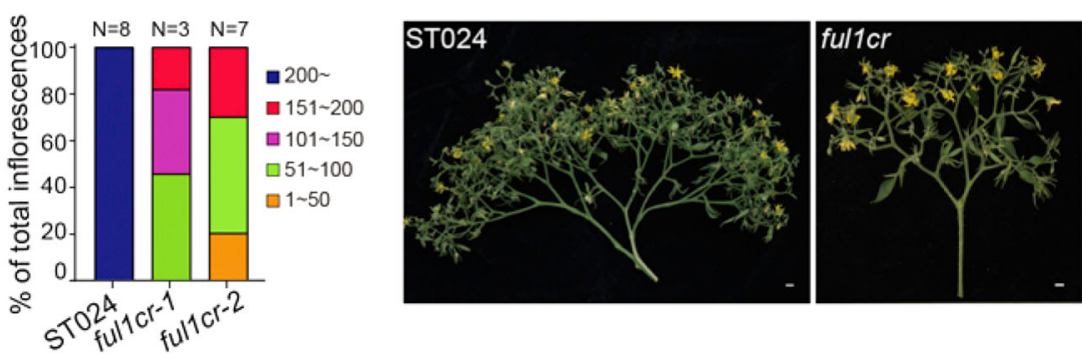

Fig. 7 FUL1 regulates inflorescence-branching development in tomato. a FUL1 mutations generated by CRISPR/Cas9. Red letters mark the position of targets. Blue letters indicate the protospacer-adjacent motif (PAM). Green letters mark the mutation sequences of ful1 mutants in ST024. b Quantification of inflorescence branching in ST024 and full cr mutants. N, plant number. c Representative inflorescence images of ST024 and ful1 cr mutants. Scale bars $=1 \mathrm{~cm}$ 
SOC1 in Arabidopsis that controls flowering ${ }^{45}$, three homologs of SOC1 are in the tomato genome (Supplementary Fig. S2a). Our results indicate that STM3 has a similar function in flowering time and that its null mutants flowered later in tomato (Supplementary Fig. S5). However, the flowering time of the $t m 3 \mathrm{cr}$ mutant was almost unaffected, suggesting that the functions of STM3 and TM3 were partly segregated in tomato. In Arabidopsis, SOC1 also functions in inflorescence architecture and acts redundantly with three other MADS-box transcription factors, SEP4, AP1, and SVP, to repress inflorescence branching. In contrast to the stm3 $\mathrm{cr}$ and $t \mathrm{~m} 3 \mathrm{cr}$ mutants showing reduced inflorescence branching, the quadruple mutant shows a massive inflorescence-branching phenotype ${ }^{30}$, indicating that STM3 and its Arabidopsis homolog play reverse functions in regulating inflorescence-branching development. Therefore, the diverse functions of STM3 imply distinct mechanisms underlying tomato and Arabidopsis inflorescence development.

As a MADS-box protein, SOC1 can directly target downstream genes by binding to the CArG box motif. Hundreds of SOC1 targets have been identified in Arabidopsis by ChIP-seq, including several genes that regulate flowering time and meristem maintenance ${ }^{28,29}$. Similarly, we found that STM3 could regulate the expression levels of flower development- and meristem maintenancerelated genes according to our RNA-seq data. In addition, it is worth noting that FUL1 is also repressed in the stm $3 \mathrm{cr}$ - 1 reproductive meristems, which was previously reported to be expressed during ripening and interact with RIN to mediate ethylene-independent aspects of fruit ripening in tomato ${ }^{46}$. We found that FUL1 is directly bound by STM3 through one of the CArG boxes on its promoter region in tomato, further supporting that FUL1 is regulated by STM3 in tomato meristems. In accordance with the role of FUL1 in inflorescence branching, FUL1 is also expressed in vegetative and reproductive meristems ${ }^{23}$, and the fullcr mutants could partially rescue the massive inflorescence branching of ST024. However, the inflorescence architectures of ful1 $\mathrm{cr}$ mutants are still more complicated than those of $s t m 3 \mathrm{cr}$ mutants, suggesting that FUL1 is one of the downstream targets of STM3 in tomato inflorescence development. In the future, the identification of more direct targets of STM3 will help us to understand the molecular mechanisms and regulatory network of inflorescence development in tomato.

\section{Materials and methods}

\section{Plant materials and growth conditions}

RILs derived from S. Lycopersicum cv. Moneymaker (MM) and S. Lycopersicum var. cerasiforme LA1310 (CC) were used as materials for the assessment of inflorescence branches as we described previously ${ }^{32}$. The NILs were generated by crossing ST147 and ST024. Then, heterozygous offspring at the qMIB1 locus were selected for selfing. After six generations, we used markers to select plants homozygous for $M I B 1^{\mathrm{CC}}$ or $m i b 1^{\mathrm{MM}}$ and to determine the introgressed region $(\sim 191 \mathrm{~kb})$ around the qMIB1 locus. Seedlings of RILs, NILs, and transgenic plants were grown in a commercial nursery for 30-40 days and then transplanted to fields. For inflorescence-branching assessment, all tomato plants were grown in a greenhouse at the Shunyi experimental station in Beijing, China. At least six individual plants of each accession were used for inflorescence-branching examination. From each plant, 5-6 inflorescences were evaluated. The $\%$ of total inflorescences represents the proportion of the number of inflorescences within a certain range to a total number of inflorescences.

\section{Fine mapping of $q M I B 1$}

An $F_{2}$ population derived from the cross between two RILs, ST147 and ST024, with the $j 2^{\text {del }} e j 2^{W}$ genotype was used for mapping. We selected 24 plants with extremely compound inflorescences (mean number of inflorescence branches $>200$ ) and 31 plants with simple inflorescences (mean number of inflorescence branches $<1.5$ ) for bulked segregant analysis (BSA). Equal amounts of tissue from each plant were pooled for DNA extraction using standard protocols. The genomic DNA was sheared using a Diagenode Bioruptor Plus instrument to obtain $\sim 300 \mathrm{bp}$ fragments. Libraries were prepared using the NEXTflex ${ }^{\mathrm{TM}}$ Rapid DNA-Seq Kit for Illumina (NOVA-5144-08) according to the manufacturer's protocol. Genomic DNA reads were trimmed by quality using Trimmomatic ${ }^{47}$, and paired reads were mapped to the reference tomato genome (SL2.50) using BWA-MEM ${ }^{48}$. SNP calling was performed as described previously ${ }^{49}$. BSA was performed with modification ${ }^{50}$. SNPs between two parental genomes were identified for further analysis when the base quality value was $\geq 20$ and the SNP quality value was $\geq 20$. On the basis of these criteria and the number of SNPs with a read depth $\geq 5$, an SNP index was calculated for both bulk samples expressing the proportion of reads harboring SNPs that were identical to those in the parent (ST147). The $\triangle$ SNP index was obtained by subtracting the SNP index for the simple inflorescence bulk sample from that for the compound inflorescence bulk sample. An average SNP index for the compound and simple inflorescence bulk samples was calculated using a $1,000 \mathrm{~kb}$ sliding window with a step size of $10 \mathrm{~kb}$. For fine mapping $q M I B 1,38$ recombinant plants were selected according to the region determined by BSA-seq from $960 \mathrm{~F}_{2}$ seedlings and self-pollinated in the greenhouse. The progeny of ten recombinant plants was selected, and their inflorescencebranching number was observed. 


\section{RNA extraction and gene expression analysis}

RNA was isolated using TRIzol Universal reagent (Tiangen, DP424). DNA contamination was removed using the TURBO DNA-free Kit (Ambion, AM1907). Reverse transcription was performed with $5 \times$ All-In-One MasterMix with AccuRT Genomic DNA Removal Kit (abm, G492) using $2 \mu \mathrm{g}$ of total RNA. Quantitative PCR (qPCR) was performed with EvaGreen $2 \times \mathrm{qPCR}$ MasterMix-No Dye (Abm, MasterMix-S) on a Bio-Rad CFX-96 Real-Time PCR instrument using the following program: $3 \mathrm{~min}$ at $95^{\circ} \mathrm{C}$ followed by 40 cycles of $20 \mathrm{~s}$ at $95^{\circ} \mathrm{C}, \quad 30 \mathrm{~s}$ at $60^{\circ} \mathrm{C}$, and $20 \mathrm{~s}$ at $72^{\circ} \mathrm{C}$. UBI3 (Solyc01g056840) was used as the internal control for qRT-PCR. The primers used for qRT-PCR are listed in Supplementary Table S4.

\section{RNA-seq analysis}

Total RNA was isolated from reproductive meristematic tissues collected from 21-d-old seedlings of ST024, tm $3 c r-1$, stm $3 c r-1$, and tm 3 stm $3 c r-1$. Three biological replicates were performed, and each biological replicate contained at least 200 individuals. A total of twelve RNASeq libraries were constructed and sequenced using Illumina HiSeq2000 at Berry Genomics (http://www. berrygenomics.com/). The filtered clean reads were aligned to the tomato genome (ITAG4.0) by STAR v2.5.3, and their features were counted by feature Counts v 1.5.3, as described in a previous paper ${ }^{51}$. The statistical package DEGseq with the MA-plot-based method in $\mathrm{R}$ version 3.0.3 was used to calculate the $P$ value, which was adjusted using the Benjamini-Hochberg procedure. The fold change between the ST024 and tm3cr-1, ST024 and stm3cr-1, and ST024 and stm 3 tm 3cr-1 libraries was calculated as FPKM (fragments per kilobase of transcript sequence per million base pairs sequenced). The thresholds for the identification of DEGs were as follows: FPKM $>1$ in any tissue, fold change $>1.5$ or $<0.6666$, and Benjamini-Hochberg adjusted $P$ value $<0.05$. GO analyses of DEGs were performed using their best homologous genes in Arabidopsis with DAVID (Database for Annotation, Visualization, and Integrated Discovery, https://david.ncifcrf.gov/).

\section{RACE assay}

A SMARTer RACE cDNA Amplification Kit (Clontech, 634923) was used for the RACE assay according to the manufacturer's instructions. Approximately $1 \mu \mathrm{g}$ of total RNA was used to prepare RACE-Ready first-strand cDNA. Then, the $3^{\prime}$ and $5^{\prime}$ ends of cDNA were amplified by using the $3^{\prime}$ or $5^{\prime}$ gene-specific primers listed in Supplementary Table S4. The obtained PCR products were ligated to vectors for sequencing.

\section{Constructs and plant transformation}

For CRISPR/Cas9 constructs, two sgRNA binding sites per gene were selected with the CRISPR-P v2.0 tool (http://cbi.hzau.edu.cn/CRISPR2/). Primers containing sgRNAs and BsaI recognition sites were used to amplify the sgRNAX_U6-26t_SIU6p_sgRNAX fragments using a pCBC_DT1T2_SlU6p vector as the template, after which the fragments were purified and cloned into pTX041 at the BsaI sites ${ }^{52,53}$. To generate 35S::STM3-YFP-HA, 35S:: TM3-YFP-HA, and 35S::STM3-GFP-FLAG constructs, the coding sequences of STM3 and TM3 were fused to the $\mathrm{N}$-terminus of YFP-HA or GFP-FLAG, respectively. All plasmids were validated by sequencing and then transformed into the Agrobacterium tumefaciens strain AGL1. The transgenic plants were validated by PCR and sequencing. All primers used are listed in Supplementary Table S4.

\section{In situ hybridization}

In situ hybridization was performed as described in Scott et al. with modifications ${ }^{54}$. The STM3 and TM3 cDNA segments were amplified with the primers P9 and P7, respectively (Supplementary Table S4), and then cloned into pEAZY-T3 (TransGen, CT301-01), which contained T7 and SP6 promoter sequences. In vitro transcription was performed with T7 RNA polymerase to produce the antisense or sense probe for in situ hybridization.

Meristem tissues were dissected by hand and fixed for $24 \mathrm{~h}$ at $4{ }^{\circ} \mathrm{C}$ in freshly prepared $4 \%(\mathrm{w} / \mathrm{v})$ paraformaldehyde buffered with phosphate-buffered saline (PBS, pH 7.2). Fixed tissues were dehydrated in a graded ethanol: histochoice (Sigma, H2779) series and impregnated with paraplast (Sigma, P3683). Dewaxed thin sections $(10 \mu \mathrm{m})$ were hybridized with hydrolysis probes for $12 \mathrm{~h}$ at $55^{\circ} \mathrm{C}$. Complete color development sections were observed using a fluorescence microscope (Leica, DM5500).

\section{Yeast two-hybrid}

Yeast two-hybrid assays were performed according to the Yeastmaker ${ }^{\mathrm{TM}}$ Yeast Transformation System 2 user manual (Clontech, PT1172-1). To explore interactions between STM3, TM3, and itself, the full-length coding sequence of each gene was cloned into bait and prey vectors, pGBKT7 and pGADT7, respectively. A pair of bait and prey plasmids were cotransformed into the $\mathrm{Y} 2 \mathrm{H}$ gold yeast strain according to the Clontech yeast protocol handbook instructions. The resultant strains were subsequently grown on plates lacking leucine and tryptophan for 3 days at $30^{\circ} \mathrm{C}$. The interaction was tested via growth assays on media lacking leucine, tryptophan, histidine, and adenine but containing X-alpha-Gal. 


\section{Co-immunoprecipitation assay}

The Co-IP assay was performed as previously described with minor modifications ${ }^{55}$. The coding sequences of STM3 and TM3 were fused to the N-terminus of FLAG or YFP-HA tags to construct 35S::STM3-FLAG, 35S::STM3$Y F P-H A$, and $35 S:: T M 3-Y F P-H A$ vectors. These plasmids were transformed into A. tumefaciens strain EHA105 and then coinfiltrated into the leaves of 4-w-old $N$. benthamiana plants together with P19. Equal numbers of samples were collected two days after infiltration, ground in liquid nitrogen, and then homogenized in $1 \mathrm{ml}$ of extraction buffer: $50 \mathrm{mM}$ Tris- $\mathrm{HCl}, \mathrm{pH} 7.5 ; 150 \mathrm{mM}$ $\mathrm{NaCl} ; 0.19 \%$ CA630; $20 \%$ glycerol; 5 mM DTT; and 1 tablet $/ 50 \mathrm{ml}$ of protease inhibitor cocktail (Roche, 04693132001). The lysates were mixed sufficiently and then centrifuged at $16,000 \times g$ for $10 \mathrm{~min}$ at $4{ }^{\circ} \mathrm{C}$. In addition, $5 \mu \mathrm{g}$ anti-HA (Sigma, H6908) antibody and $20 \mu \mathrm{l}$ Dynabeads Protein G (Novex, 10001D) were incubated for $2 \mathrm{~h}$ at $4{ }^{\circ} \mathrm{C}$. The lysates and the HA-binding beads were incubated overnight at $4{ }^{\circ} \mathrm{C}$. The incubated beads were washed five times with $1 \times \mathrm{PBS}$. Then, $30 \mu \mathrm{l}$ of $6 \times$ protein loading buffer was added and boiled for $5 \mathrm{~min}$. The proteins were electrophoretically separated by $10 \%$ SDS-PAGE and transferred to a PVDF membrane (Immobilon-P, IPVH00010). Immunoblots were performed using an anti-FLAG antibody (MBL, M185-3L, 1:2000) for STM3-FLAG. The bands were visualized on a Tanon-5200 Chemiluminescent Imaging System (Tanon Science and Technology).

\section{ChIP and ChIP-qPCR}

ChIP was performed using $0.5 \mathrm{~g}$ hand-dissected meristems of 18-d-old seedlings of 35S::STM3-YFP-HA transgenic plants as described previously with minor modifications ${ }^{32}$. Meristems were completely ground in liquid nitrogen and cross-linked in $1 \%$ formaldehyde (Sigma-Aldrich) for $10 \mathrm{~min}$ at $4{ }^{\circ} \mathrm{C}$. Chromatin was sheared using a Diagenode Bioruptor Plus instrument to obtain 300 bp DNA fragments. Anti-HA (Sigma, H6908) was used for immunoprecipitation. The DNA isolated by ChIP was used for qPCR analysis or Illumina paired-end sequencing. qPCR was performed using EvaGreen $2 \times \mathrm{qPCR}$ MasterMix-No Dye (Abm, MasterMix-S) on a Bio-Rad CFX-96 Real-Time PCR instrument with the following program: $3 \mathrm{~min}$ at $95^{\circ} \mathrm{C}$ followed by 50 cycles of $20 \mathrm{~s}$ at $95^{\circ} \mathrm{C}, 30 \mathrm{~s}$ at $60^{\circ} \mathrm{C}$, and $20 \mathrm{~s}$ at $72^{\circ} \mathrm{C}$. The intergenic region around ACTIN (Solyc03g078400) was used as an internal control. Primers for qPCR are listed in Supplementary Table S4.

\section{Electrophoretic mobility shift assay}

The full-length coding regions of STM3 were amplified by PCR using the primer pair in Supplementary Table S4. The PCR product was ligated into the MBP-pMCSG7 plasmid containing a polyhistidine $(6 \times \mathrm{His})$ sequence by a ligation-independent cloning method as previously described $^{56}$. All recombinant proteins were expressed and purified from Escherichia coli strain BL21 RIL (BL21 CP, Stratagene). The MBP-STM3 protein and MBP protein were induced by $0.2 \mathrm{mM}$ isopropylthio-b-D-galactoside at $16^{\circ} \mathrm{C}$ for $16 \mathrm{~h}$. Ni-NTA agarose (QIAGEN, 1018244) was used to purify MBP-STM3 and MBP according to the manufacturer's instructions. We synthesized and annealed the $5^{\prime}$ biotin DNA probes used in EMSA. DNA gel shift assays were performed using the LightShift Chemiluminescent EMSA kit (Thermo Fisher Scientific, 20148). Each EMSA binding reaction $(20 \mu \mathrm{L})$ contained $0.5 \mu \mathrm{L}$ purified recombinant protein, $4 \mu \mathrm{L}$ biotin-labeled probe DNA (100 pmol), $2 \mu \mathrm{L}$ binding buffer, and $1 \mu \mathrm{L}$ poly (dI-dC). Transferred DNA and protein were cross-linked using a UV lamp at $312 \mathrm{~nm}$. The biotin-labeled DNA was determined using a Thermo Scientific chemiluminescence kit. The bands were visualized on a Tanon-5200 Chemiluminescent Imaging System (Tanon Science and Technology).

\section{Dual-luciferase reporter assay}

For plasmid construction, the pluc-35Rluc backbone vector was obtained from PPZP211 ${ }^{57}$. The nearly $2.3 \mathrm{~kb}$ FUL1 promoter sequence was amplified using $\mathrm{MM}$ genomic DNA as a template and integrated into pluc35Rluc using the in-fusion HD cloning kit (Clontech, 639649). Then, the mutated FUL1 promoter was generated by deleting the $40 \mathrm{bp}$ sequence including the CArG box in the $\mathrm{S} 1$ region of the promoter (Fig. $6 \mathrm{~b}$ ). The primers used for the constructs are listed in Supplementary Table S4. The plasmids were transformed into Agrobacterium tumefaciens EHA105 competent cells. A single colony was cultured in Luria-Bertani (LB) medium until the $\mathrm{OD}_{600}$ value reached 1 . The $A$. tumefaciens cells were collected by centrifugation and suspended using $10 \mathrm{mM}$ $\mathrm{MgCl}_{2}$ and $150 \mu \mathrm{M}$ acetosyringone. The cells containing the overexpression plasmids, luciferase plasmid, and p19 plasmid were mixed in a volume ratio of 2:1:3 and infiltrated into Nicotiana benthamiana leaves using a syringe. The leaves were harvested and ground in liquid nitrogen at $2 \mathrm{~d}$ after infiltration. The activities of firefly luciferase and Renilla luciferase were measured using a dualluciferase reporter assay system (Promega; E1910) on a Promega GLOMAX 20/20 LUMINOMETER. Thirtyseven sets of data were collected from the different leaves. The measured data (LUC/REN) were normalized to the control on the same leaf.

\section{Acknowledgements}

This work was supported by the National Natural Science Foundation of China Grant (31930099), the National Key Research and Development Program of China (2018YFD1000801), and the Science and Technology Innovation Program of the Chinese Academy of Agricultural Sciences. 


\section{Author contributions}

X.W. carried out most of the experiments; Z.L. analyzed the RNA-seq and BSA-seq data. S. S., J.W., R. L., and H. W helped to observe the phenotype of the RIL population; X. C. conceived the project and designed the research. X.W. and X. C. wrote the paper.

\section{Data availability}

The RNA sequencing datasets generated in this study have been deposited in the Sequence Read Archive (SRA) under the accession number PRJNA706044. Other data supporting our findings are available in the manuscript file or from the corresponding author upon request.

\section{Competing interests}

The authors declare no competing interests.

Supplementary information The online version contains supplementary material available at https://doi.org/10.1038/s41438-021-00677-x.

Received: 6 March 2021 Revised: 19 July 2021 Accepted: 2 August 2021 Published online: 01 December 2021

\section{References}

1. Wang, B., Smith, S. M. \& Li, J. Genetic regulation of shoot architecture. Annu Rev. Plant Biol. 69, 437-468 (2018).

2. Welty, N., Radovich, C., Meulia, T. \& van der Knaap, E. Inflorescence development in two tomato species. Can. J. Bot. 85, 111-118 (2007).

3. Lippman, Z. B. et al. The making of a compound inflorescence in tomato and related nightshades. PLOS Biol. 6, e288 (2008).

4. Teo, Z. W., Song, S., Wang, Y. Q., Liu, J. \& Yu, H. New insights into the regulation of inflorescence architecture. Trends Plant Sci. 19, 158-165 (2014).

5. Clark, S. E., Williams, R. W. \& Meyerowitz, E. M. The CLAVATA1 gene encodes a putative receptor kinase that controls shoot and floral meristem size in Arabidopsis. Cell 89, 575-585 (1997).

6. Fletcher, J. C., Brand, U., Running, M. P., Simon, R. \& Meyerowitz, E. M. Signaling of cell fate decisions by CLAVATA3 in Arabidopsis shoot meristems. Science 283, 1911-1914 (1999).

7. Jeong, S., Trotochaud, A. E. \& Clark, S. E. The Arabidopsis CLAVATA2 gene encodes a receptor-like protein required for the stability of the CLAVATA1 receptor-like kinase. Plant Cell 11, 1925-1934 (1999).

8. Schoof, $\mathrm{H}$. et al. The stem cell population of Arabidopsis shoot meristems is maintained by a regulatory loop between the CLAVATA and WUSCHEL genes. Cell 100, 635-644 (2000).

9. Ohyama, K., Shinohara, H., Ogawa-Ohnishi, M. \& Matsubayashi, Y. A glycopeptide regulating stem cell fate in Arabidopsis thaliana. Nat. Chem. Biol. 5, 578-580 (2009).

10. Stahl, Y. \& Simon, R. Plant primary meristems: shared functions and regulatory mechanisms. Curr. Opin. Plant Biol. 13, 53-58 (2010).

11. Pautler, M., Tanaka, W., Hirano, H. Y. \& Jackson, D. Grass meristems I: shoot apical meristem maintenance, axillary meristem determinacy, and the floral transition. Plant Cell Physiol. 54, 302-312 (2013).

12. $\mathrm{Xu}, \mathrm{C}$. et al. A cascade of arabinosyltransferases controls shoot meristem size in tomato. Nat. Genet. 47, 784-792 (2015)

13. Rodriguez-Leal, D. et al. Evolution of buffering in a genetic circuit controlling plant stem cell proliferation. Nat. Genet. 51, 786-792 (2019).

14. Molinero-Rosales, N., Latorre, A., Jamilena, M. \& Lozano, R. SINGLE FLOWER TRUSS regulates the transition and maintenance of flowering in tomato. Planta 218, 427-434 (2004).

15. Lifschitz, E. et al. The tomato FT ortholog triggers systemic signals that regulate growth and flowering and substitute for diverse environmental stimuli. Proc. Natl Acad. Sci. USA 103, 6398-6403 (2006).

16. Quinet, M. et al. Characterization of tomato (Solanum Lycopersicum L.) mutants affected in their flowering time and in the morphogenesis of their reproductive structure. J. Exp. Bot. 57, 1381-1390 (2006).

17. Szymkowiak, E. J. \& Irish, E. E. Interactions between jointless and wild-type tomato tissues during development of the pedicel abscission zone and the inflorescence meristem. Plant Cell 11, 159-175 (1999).
18. Thouet, J., Quinet, M., Lutts, S., Kinet, J. M. \& Perilleux, C. Repression of floral meristem fate is crucial in shaping tomato inflorescence. PLoS One 7, e31096 (2012).

19. Molinero-Rosales, N. et al. FALSIFLORA, the tomato orthologue of FLORICAULA and LEAFY, controls flowering time and floral meristem identity. Plant J.: Cell Mol. Biol. 20, 685-693 (1999).

20. Zheng, H. \& Kawabata, S. Identification and validation of new alleles of FALSIFLORA and COMPOUND INFLORESCENCE genes controlling the number of branches in tomato inflorescence. Int. J. Mol. Sci. https://doi.org/10.3390/ ijms18071572 (2017).

21. MacAlister, C. A. et al. Synchronization of the flowering transition by the tomato TERMINATING FLOWER gene. Nat. Genet. 44, 1393-1398 (2012).

22. Xu, C., Park, S. J., Van Eck, J. \& Lippman, Z. B. Control of inflorescence architecture in tomato by BTB/POZ transcriptional regulators. Genes Dev. 30, 2048-2061 (2016)

23. Park, S. J., Jiang, K., Schatz, M. C. \& Lippman, Z. B. Rate of meristem maturation determines inflorescence architecture in tomato. Proc. Natl Acad. Sci. USA 109, 639-644 (2012).

24. Soyk, S. et al. Bypassing negative epistasis on yield in tomato imposed by a domestication gene. Cell 169, 1142-1155 e1112 (2017).

25. Soyk, S. et al. Duplication of a domestication locus neutralized a cryptic variant that caused a breeding barrier in tomato. Nat. Plants 5, 471-479 (2019).

26. Alonge, $\mathrm{M}$. et al. Major impacts of widespread structural variation on gene expression and crop improvement in tomato. Cell 182, 145-161 e123 (2020).

27. Lee, J. \& Lee, I. Regulation and function of SOC1, a flowering pathway integrator. J. Exp. Bot. 61, 2247-2254 (2010).

28. Immink, R. G. et al. Characterization of SOC1's central role in flowering by the identification of its upstream and downstream regulators. Plant Physiol. 160 433-449 (2012).

29. Tao, Z. et al. Genome-wide identification of SOC1 and SVP targets during the floral transition in Arabidopsis. Plant J.: Cell Mol. Biol. 70, 549-561 (2012).

30. Liu, C. et al. A conserved genetic pathway determines inflorescence architecture in Arabidopsis and rice. Dev. Cell 24, 612-622 (2013).

31. Reynard, G. B. New source of the j2 gene governing jointless pedicel in tomato. Science 134, 2102 (1961).

32. Zhang, S. et al. Enhancer-promoter interaction of SELF PRUNING 5 G shapes photoperiod adaptation. Plant Physiol. 178, 1631-1642 (2018).

33. Lee, $\mathrm{H}$. et al. The AGAMOUS-LIKE 20 MADS domain protein integrates floral inductive pathways in Arabidopsis. Genes Dev. 14, 2366-2376 (2000).

34. Leseberg, C. H. et al. Interaction study of MADS-domain proteins in tomato. J. Exp. Bot. 59, 2253-2265 (2008).

35. Irish, V. F. The flowering of Arabidopsis flower development. Plant J.: Cell Mol. Biol. 61, 1014-1028 (2010).

36. Ferrandiz, C., Gu, Q., Martienssen, R. \& Yanofsky, M. F. Redundant regulation of meristem identity and plant architecture by FRUITFULL, APETALA1, and CAULIFLOWER. Development 127, 725-734 (2000).

37. Teper-Bamnolker, P. \& Samach, A. The flowering integrator FT regulates SEPALLATA3 and FRUITFULL accumulation in Arabidopsis leaves. Plant Cell 17 2661-2675 (2005).

38. Bemer, M. et al. FRUITFULL controls SAUR10 expression and regulates Arabidopsis growth and architecture. J. Exp. Bot. 68, 3391-3403 (2017).

39. Balanza, V. et al. Genetic control of meristem arrest and life span in Arabidopsis by a FRUITFULL-APETALA2 pathway. Nat. Commun. 9, 565 (2018).

40. Fornes, O. et al. JASPAR 2020: update of the open-access database of transcription factor binding profiles. Nucleic Acids Res. 48, D87-D92 (2020).

41. Lee, S., Kim, J., Han, J. J., Han, M. J. \& An, G. Functional analyses of the flowering time gene OSMADS50, the putative SUPPRESSOR OF OVEREXPRESSION OF CO 1/AGAMOUS-LIKE 20 (SOC1/AGL20) ortholog in rice. Plant J.: Cell Mol. Biol. 38, 754-764 (2004).

42. Zhong, $\mathrm{X}$. et al. Cloning and expression analysis of GmGAL1, SOC1 homolog gene in soybean. Mol. Biol. Rep. 39, 6967-6974 (2012).

43. $\mathrm{Na}, \mathrm{X}$. et al. Cloning and functional analysis of the flowering gene GmSOC1-like, a putative SUPPRESSOR OF OVEREXPRESSION CO1/AGAMOUS-LIKE 20 (SOC1/AGL20) ortholog in soybean. Plant Cell Rep. 32 1219-1229 (2013).

44. Alter, P. et al. Flowering time-regulated genes in maize include the transcription factor ZmMADS1. Plant Physiol. 172, 389-404 (2016).

45. Samach, A. et al. Distinct roles of CONSTANS target genes in reproductive development of Arabidopsis. Science 288, 1613-1616 (2000). 
46. Bemer, M. et al. The tomato FRUITFULL homologs TDR4/FUL1 and MBP7/ FUL2 regulate ethylene-independent aspects of fruit ripening. Plant Cell $\mathbf{2 4}$ 4437-4451 (2012).

47. Bolger, A. M., Lohse, M. \& Usadel, B. Trimmomatic: a flexible trimmer for Illumina sequence data. Bioinformatics 30, 2114-2120 (2014).

48. Li, H. et al. The sequence alignment/map format and SAMtools. Bioinformatics 25, 2078-2079 (2009).

49. Zhang, S. et al. Detection of major loci associated with the variation of 18 important agronomic traits between Solanum pimpinellifolium and cultivated tomatoes. Plant J.: Cell Mol. Biol. 95, 312-323 (2018).

50. Lin, T. et al. Genomic analyses provide insights into the history of tomato breeding. Nat. Genet. 46, 1220-1226 (2014).

51. Chen, S. et al. Genome editing to integrate seed size and abiotic stress tolerance traits in Arabidopsis reveals a role for DPA4 and SOD7 in the regulation of inflorescence architecture. Int. J. Mol. Sci. https://doi.org/10.3390/ ijms20112695 (2019).
52. Deng, L. et al. Efficient generation of pink-fruited tomatoes using CRISPR Cas9 system. J. Genet Genomics 45, 51-54 (2018).

53. Song, J. et al. Variations in both FTL1 and SP5G, two tomato FT paralogs, control day-neutral flowering. Mol. Plant 13, 939-942 (2020).

54. Liu, X. et al. AGAMOUS terminates floral stem cell maintenance in Arabidopsis by directly repressing WUSCHEL through recruitment of Polycomb Group proteins. Plant Cell 23, 3654-3670 (2011).

55. Meng, Y., Li, H., Wang, Q., Liu, B. \& Lin, C. Blue light-dependent interaction between cryptochrome2 and CIB1 regulates transcription and leaf senescence in soybean. Plant Cell 25, 4405-4420 (2013).

56. Eschenfeldt, W. H., Lucy, S., Millard, C. S., Joachimiak, A. \& Mark, I. D. A family of LIC vectors for high-throughput cloning and purification of proteins. Methods Mol. Biol. 498, 105-115 (2009).

57. Hajdukiewicz, P., Svab, Z. \& Maliga, P. The small, versatile pPZP family of Agrobacterium binary vectors for plant transformation. Plant Mol. Biol. 25, 989-994 (1994). 